\title{
Weak and Strong Solutions of the Navier-Stokes Initial Value Problem
}

\author{
By \\ Yoshikazu GIGA*
}

\begin{abstract}
This paper reviews the existence, uniqueness and regularity of weak and strong solutions of the Navier-Stokes system. For this purpose we emphasize semigroup theory and the theory of the Stokes operator. We use dimensional analysis to clarify the meaning of the results for the solutions.
\end{abstract}

\section{§0. Introduction}

Let $D$ be a bounded domain in $\boldsymbol{R}^{n}(n \geq 2)$ with smooth boundary $S$. We consider the initial-boundary value problem for the Navier-Stokes equations

$$
\begin{gathered}
\partial u / \partial t-\Delta u+(u, \operatorname{grad}) u+\operatorname{grad} p=f, \operatorname{div} u=0 \text { in } D \times(0, T), \\
u=0 \text { on } S \times(0, T), \quad u(x, 0)=a(x) \text { in } D,
\end{gathered}
$$

where $(u, \operatorname{grad})=\sum_{j=1}^{n} u^{j}\left(\partial / \partial x_{j}\right)$. This system describes the motion of viscous incompressible fluid filling a rigid vessel $D$. The function $u=\left(u^{1}(x, t)\right.$, $\left.\ldots, u^{n}(x, t)\right)$ represents the velocity of the fluid and $p(x, t)$ is the pressure. The function $a=\left(a^{1}(x), \ldots, a^{n}(x)\right)$ is a given initial velocity and $f=\left(f^{1}(x, t), \ldots, f^{n}(x\right.$, t)) is a given external force.

We discuss the existence, uniqueness and regularity of weak and strong solutions of this problem. There is an extensive literature on this subject since Leray [27-29] introduced many useful and fundamental ideas. In [29] he constructed a global (in time) weak solution and a local strong solution of the initial value problem when $D=\boldsymbol{R}^{3}$. Hopf [20] has proved the existence of a global weak solution of the initial-boundary value problem. Such weak solu-

Received February 1, 1983.

* Courant Institute of Mathematical Sciences, New York University, 251 Mercer Street, New York, N. Y. 10012, U. S. A.

* On leave from Department of Mathematics, Nagoya University, Nagoya 464, Japan.

* Partly supported by Sakkokai Foundation. 
tions are called Leray-Hopf solutions. Since then, many mathematicians studied the uniqueness and regularity of Leray-Hopf solutions. It turns out that if the space dimension $n$ is two, Leray-Hopf solutions are unique and regular; see Lions-Prodi [31], Lions [30], Ladyzhenskaya [26], Serrin [38] and Temam [43]. However, for $n \geq 3$ the uniqueness and regularity of Leray-Hopf solutions are still open problems.

There are many contributions to the theory of more regular solutions, namely, strong solutions; see Itô [21], Kiselev and Ladyzhenskaya [24], Kato and Fujita [10, 23],..., see also Ladyzhenskaya [26], Serrin [38] and Temam [43] and papers cited there. However, up to now the global existence of strong solution has been proved only when the data $a$ and $f$ are sufficiently small.

Many tools in functional analysis and the theory of partial differential equations are used to prove the existence, uniqueness and regularity of solutions. The energy estimate for solutions is fundamental to prove that there is a global weak solution. However, every method has advantages and disadvantages. If we discuss the existence of a unique local strong solution, the semigroup method introduced by Kato and Fujita [10,23] and Sobolevskii [39] is more powerful than the energy estimate. Recently the semigroup method is strengthened by Giga and Miyakawa [16]; see Giga [18] for the summary. If we use the semigroup method to construct solutions, we need less regularity of the initial data $a$.

This paper intends to review the existence, uniqueness and regularity theory. We emphasize the theory of the Stokes operator and semigroup theory to clarify what results heavily depend on the energy estimate. It turns out that many nice results can be proved if we use the theory of the Stokes operator in $L_{p}$ spaces $(1<p<\infty)$ which is given in $[14,15]$. Although the results in this paper are known results or just their combinations, I believe the proofs given here are conceptually simple and easy to understand. There are many good review articles (eg. [26, 38, 43, 44]). However, few of them include the semigroup approach, so some parts of arguments seem to be complicated.

Another purpose of this paper is to explain the difference between the cases $n=2$ and $n \geq 3$. The energy estimate brings many a priori estimates. When $n=2$, the energy estimate is strong enough to prove the global existence of smooth solution. However, when $n \geq 3$, the energy estimate is not so strong. If $n=3$, we can estimate the size of possible singular set of Leray-Hopf solutions, using the energy estimate. The first contribution to this direction is due to Leray [29]. Later, Scheffer [32-35] estimated Hausdorff dimension of the singular 
set, introducing a clever technique. Recently, Caffarelli, Kohn and Nirenberg [3] improve his results; see also Kohn [25] for the summary. In [3] dimensional analysis is introduced to explain their theory. Here we use dimensional analysis to clarify the difference between the cases $n=2$ and $n \geq 3$.

In the first three sections we recall properties of the Stokes operator and estimates for the nonlinear term in (NS). Conceptually, the results in these sections are easy to understand, although the proofs are not so easy; see $[14,15$, $16,41]$.

In Section 4 we discuss a priori estimates derived from the energy estimate.

In Section 5 we state the theorems for weak solutions. We are more interested in the uniqueness than the existence.

In Section 6 we construct strong solutions, using semigroup theory.

In Section 7 we study the regularity of Leray-Hopf solutions. Here we use the energy estimate, strong solutions and the uniqueness theorem.

I try to give proofs or ideas of proofs as far as possible. However, I do not intend to make an exhaustive presentation of the recent results nor to give a complete list of references.

I am grateful to Professor Robert Kohn for useful discussions. I am also grateful to Dr. Zensho Yoshida for reading the manuscript of this paper.

\section{$\S 1$. Function Spaces}

We begin with recalling the Helmholtz decomposition of vector fields which is frequently used in the theory of the Navier-Stokes equations.

Let $D$ be a domain in $\boldsymbol{R}^{n}$. For $p \geq 1$ let $L_{p}(D)$ be the space of complex valued measurable functions on $D$ with integrable $p$-th power. This space is a Banach space equipped with the usual norm

$$
|u|_{p}=\left(\int_{D}|u(x)|^{p} d x\right)^{1 / p}
$$

The space $L_{2}(D)$ is a Hilbert space endowed with the usual inner product

$$
(u, v)=\int_{D} u(x) \overline{v(x)} d x .
$$

We denote by $H_{p}^{m}(D)$ the Sobolev space of functions which are in $L_{p}(D)$ together with all their derivatives of order $\leq m$. The reader is refered to Adams [1] for the theory of Sobolev spaces. 
In what follows we assume that $D$ is a bounded domain with smooth boundary $S$ and that $1<p<\infty$. Set

$$
\begin{aligned}
& X_{p}=\text { the closure in }\left(L_{p}(D)\right)^{n} \text { of }\left\{u \in\left(C_{0}^{\infty}(D)\right)^{n} ; \operatorname{div} u=0\right\}, \\
& G_{p}=\left\{\operatorname{grad} q ; q \in H_{p}^{1}(D)\right\} ;
\end{aligned}
$$

here $C_{0}^{\infty}(D)$ denotes the space of smooth functions with compact support in $D$. We then have the Helmholtz decomposition

$$
\left(L_{p}(D)\right)^{n}=X_{p} \oplus G_{p} \quad(\text { direct sum }) .
$$

When $p=2$, this is the orthogonal decomposition discussed in standard literature, for example Ladyzhenskaya [26] and Temam [43]. However, for general $p$ this result may not widely be known; for the proof see Fujiwara and Morimoto [13].

We can describe $X_{p}$ explicitly. To do this we explain the continuous projection $P$ onto $X_{p}$ associated with this decomposition. Consider the Neumann problem

$$
\Delta q=\operatorname{div} f \text { in } D,(\partial / \partial v) q=f \cdot v \text { on } S,
$$

where $v$ denotes the unit interior normal vector to $S$. Heuristically, $P$ can be defined by

$$
P f=f-\operatorname{grad} q \text {. }
$$

(This is not precise because the trace $f \cdot v$ is not defined for general $f \in\left(L_{p}(D)\right)^{n}$ for the precise definition of $P$; see Fujiwara and Morimoto [13].) This gives

$$
X_{p}=\left\{u \in\left(L_{p}(D)\right)^{n} ; \operatorname{div} u=0 \text { in } D, u \cdot v=0 \text { on } S\right\} .
$$

When $p=2, X_{2}$ is a Hilbert space, so we denote it by $H$.

\section{§ 2. The Stokes Operator}

To study the linear part of the Navier-Stokes equations we introduce the Stokes operator.

Let $D$ be a bounded domain in $\boldsymbol{R}^{n}$ with smooth boundary $S$. We consider the Stokes problem

$$
-\Delta u+\operatorname{grad} p=f, \quad \operatorname{div} u=0 \quad \text { in } D, \quad u=0 \text { on } S .
$$

The Stokes operator $A$ in $X_{p}$ is defined by $A=-P \Delta$ with dense domain

$$
D(A)_{p}=X_{p} \cap\left\{u \in\left(H_{p}^{2}(D)\right)^{n} ; u=0 \text { on } S\right\},
$$


where $1<p<\infty$. Applying $P$ to both sides of the Stokes equations, we get

$$
A u=P f \quad \text { in } X_{p},
$$

since $P(\operatorname{grad} p)=0$.

The Stokes operator $A$ has many properties resembling to those of the Laplace operator. For example, Cattabriga [5] showed that $A$ has a bounded inverse in $X_{p}$. His proof is for $n=3$, but can be generalized for general $n$; see also Agmon, Douglis and Nirenberg [2], Solonnikov [40] and Vorovich and Yudovich [46]. This in particular implies that $\mathrm{A}$ is a closed linear operator. When $p=2, A$ is a positive self-adjoint linear operator defined on $\mathrm{H}_{2} \mathrm{X}_{2}$; here, positive means

$$
(A u, u)>0 \quad \text { for } \quad u \in D(A)_{2}, \quad u \neq \equiv 0 .
$$

Remark. The Stokes operator is not equal to the Laplace operator; in the definition of $A$ the projection $P$ is necessary. Indeed, $A u$ is not in $X_{p}$ for general $u \in D(A)_{p}$, because we cannot expect that $\Delta u \cdot v$ identically vanishes on $S$. If we consider the Stokes operator on Riemannian manifold without boundary we can omit $P$ in the definition of $A$, because $\Delta$ commutes with div. In this situation the Stokes operator is equal to the Laplace operator. Simplest examples of such manifolds are $n$-dimensional Euclidian space and $n$-dimensional flat torus. Obviously, to consider the latter is the same to give the periodic boundary condition on a cube, which is discussed in Temam [44].

We now write the Navier-Stokes equations as an evolution equation in $X_{p}$, using the Stokes operator $A$. Applying $P$ to both sides of (NS) yields

$$
d u / d t+A u+B u=P f \quad(t>0), \quad u(0)=a,
$$

where $B u=P(u$, grad $) u$; we assume here $P a=a$.

In what follows we study (l) instead of (NS). As far as $u$ is sufficiently smooth (I) is equivalent to (NS).

We first study the linear homogeneous part of this equation, i.e.,

$$
d v / d t+A v=0(t>0), \quad v(0)=a .
$$

The solution $v$ is expressed by $v=e^{-t A} a$. Here $e^{-t A}$ is called the semigroup generated by $-A$. For $e^{-t A}$ we have

Lemma 2.1 ([14], Solonnikov [41]). The semigroup $e^{-t A}$ is a bounded holomorphic semigroup in $X_{p}$. That is, for every nonnegative integer $m$ the estimate 


$$
\left|A^{m} e^{-t A} f\right|_{p} \leq C t^{-m}|f|_{p}, \quad f \in X_{p}, t>0
$$

is valid with constant $C$.

Conceptually, "holomorphic" means that the solution $v=e^{-t A} a$ is holomorphic in time. This is a typical property of parabolic equations so we sometimes say parabolic semigroup instead of holomorphic semigroup; for the details of holomorphic semigroup, see Tanabe [42] and Yosida [47].

The proof of Lemma 2.1 for general $p$ is complicated. However, when $p=2$, the proof is easy so we give it here. To prove that $e^{-t A}$ is bounded holomorphic in $\mathrm{H}=\mathrm{X}_{2}$ it is enough to show

$$
\left|(\lambda+A)^{-1} f\right|_{2} \leq C(\varepsilon)|\lambda|^{-1}|f|_{2}, \quad f \in H,|\arg \lambda| \leq \pi-\varepsilon,
$$

for some $0<\varepsilon<\pi / 2$; see Tanabe [42]. Since $(A u, u)>0$, we have

$$
|((\lambda+A) u, u)|=\left.|\lambda| u\right|_{2} ^{2}+(A u, u)|\geq| \operatorname{Im} \lambda \|\left. u\right|_{2} ^{2} \geq|\lambda| \sin \varepsilon|u|_{2}^{2} .
$$

Using the Schwarz inequality to the left hand side gives

$$
|(\lambda+A) u|_{2}|u|_{2} \geq|\lambda| \sin \varepsilon|u|_{2}^{2}
$$

which completes the proof.

Since $A^{-1}$ exists, Lemma 2.1 enables us to define fractional power $A^{\alpha}$ for $\alpha \in R$; see Tanabe [42] and Yosida [47]. Lemma 2.1 now implies

Lemma 2.2. For every positive $\alpha$ the estimate

$$
\left|A^{\alpha} e^{-t A} f\right|_{p} \leq C t^{-\alpha}|f|_{p}, \quad t>0
$$

is valid for all $f$ in $X_{p}$.

We now study $A^{\alpha}$. Let $V_{p}^{2 \alpha}$ be the domain of $A^{\alpha}$ in $X_{p}$, i.e., $V_{p}^{2 \alpha}=D\left(A^{\alpha}\right)_{p}$. This is a Banach space equipped with the norm $\left|A^{\alpha} u\right|_{p}$. We can characterize $V_{p}^{2 \alpha}$ using complex interpolation space; for the definition see Calderón [4]. Let $H_{p}^{2 \alpha}(D)$ be the space of Bessel potentials, i.e., for $0 \leq \alpha \leq 1 H_{p}^{2 \alpha}(D)$ is defined by the complex interpolation space $\left[L_{p}(D), H_{p}^{2}(D)\right]_{\alpha}$; see Adams $[1]$.

Lemma 2.3 ([15]). We have

$$
V_{p}^{2 \alpha}=\left[X_{p}, V_{p}^{2}\right]_{\alpha}, \quad 0 \leq \alpha \leq 1 .
$$

In particular $V_{p}^{2 \alpha}$ is continuously embedded in $H_{p}^{2 \alpha}$ for $\alpha \geq 0$. Moreover, we have $V_{p}^{1}=X_{p} \cap\left\{u \in\left(H_{p}^{1}(D)\right)^{n} ; u=0\right.$ on $\left.S\right\}$.

Remark. For the Laplace operator the corresponding properties are known 
by Fujiwara [12] and Seeley [36]. For $p=2, V_{p}^{2 \alpha}$ is characterized by Fujita and Morimoto [11].

Definition of complex interpolation shows the following interpolation inequalities; see Calderón [4]. For $0<\alpha<\sigma<\beta$ the estimate

$$
\left|A^{\sigma} u\right|_{p} \leq C\left|A^{\alpha} u\right|_{p}^{\gamma}\left|A^{\beta} u\right|_{p}^{\delta}, \quad \gamma+\delta=1, \alpha \gamma+\beta \delta=\sigma,
$$

is valid for all $u$ in $V_{p}^{2 \beta}$.

\section{§3. Nonlinear Term}

In this section we study the nonlinear term $B u=P(u$, grad $) u$. We begin with recalling the well known properties of the tri-linear form

$$
b(u, v, w)=((u, \operatorname{grad}) v, w)=\int_{D}(u, \operatorname{grad}) v \cdot w d x .
$$

Here functions are arbitrary as far as following calculations are meaningful.

Lemma 3.1. If $u$ is divergence free and $u \cdot v$ identically vanishes on $S$, then we have

$$
b(u, v, w)=-b(u, w, v) .
$$

In particular, we have

$$
b(u, v, v)=0 .
$$

Proof. Definition of $b$ gives

$$
b(u, v, w)=\int_{D} \sum_{i, j=1}^{n}\left(u^{i} \frac{\partial}{\partial x_{i}} v^{j}\right) w^{j} d x .
$$

Since $\left(\partial / \partial x_{i}\right)\left(u^{i} v^{j}\right)=u^{i}\left(\partial / \partial x_{i}\right) v^{j}+v^{j}\left(\partial / \partial x_{i}\right) u^{i}$, the right hand side is equal to

$$
\int_{D} \sum_{i, j=1}^{n}\left(\frac{\partial}{\partial x_{i}}\left(u^{i} v^{j}\right)\right) w^{j} d x-\sum_{j=1}^{n} \int_{D}(\operatorname{div} u) v^{j} w^{j} d x .
$$

Since $\operatorname{div} u=0$, the second term disappears. Integrating the first term by parts yields

$$
-\int_{D} \sum_{i, j=1}^{n} u^{i} v^{i} \frac{\partial}{\partial x_{i}} w^{j} d x-\int_{S} \sum_{j=1}^{n}(u \cdot v) v^{j} w^{j} d S
$$

Since $u \cdot v=0$ on $S$, the second term vanishes. The first term is equal to $-b(u$, $w, v)$, so we have $b(u, v, w)=-b(u, w, v)$, which completes the proof. 
We estimate the nonlinear term, using the Sobolev inequality and Lemma 2.3. The Sobolev inequality we use here is

$$
|f|_{r} \leq C\|f\|_{H_{p}^{\beta}} \quad \text { for all } f \text { in } \quad H_{p}^{\beta}(D) \quad(\beta \geq 0)
$$

such that $1 / r \geq 1 / p-\beta / n>0$; see Adams [1]. Here $H_{p}^{\beta}(D)$ is the space of Bessel potentials defined in Section 2, so $\beta$ is not necessarily an integer. Combine Lemma 2.3 and the above inequality to get

$$
|f|_{r} \leq C^{\prime}\left|A^{\beta / 2} f\right|_{p} \quad \text { for all } f \text { in } V_{p}^{\beta},
$$

which is a key to estimate the nonlinear term.

Using this, we now give the estimates for $P(u, \operatorname{grad}) v$.

Lemma 3.2 ([16]; see also $[10,23]$ for $p=2)$. Let $0 \leq \delta<1 / 2+n(1-1 / p) / 2$. We have

$$
\left|A^{-\delta} P(u, \operatorname{grad}) v\right|_{p} \leq M\left|A^{\theta} u\right|_{p}\left|A^{\rho} v\right|_{p}
$$

with a constant $M=M(\delta, \theta, \rho, p)$ if $\delta+\theta+\rho \geq n / 2 p+1 / 2, \theta>0, \rho>0, \rho+\delta>1 / 2$. In particular, if $p=n$, we can choose $\delta=1 / 4, \theta=1 / 4, \rho=1 / 2$.

This Lemma includes many kinds of estimates for $P(u, \operatorname{grad}) v$ as a particular case. For example, when $n=p=2$, we have

$$
|P(u, \operatorname{grad}) v| \leq C|u|^{1 / 2}\left|A^{1 / 2} u\right|^{1 / 2}\left|A^{1 / 2} v\right|^{1 / 2}|A v|^{1 / 2} ;
$$

see Temam [44]. Here $|u|$ denotes $L_{2}$-norm of $u$. Indeed, Lemma 3.2 with $n=p=2, \delta=0$ gives

$$
|P(u, \operatorname{grad}) v| \leq M\left|A^{1 / 4} u\right|\left|A^{3 / 4} v\right| .
$$

Applying the interpolation inequalities in Section 2, we have

$$
\left|A^{1 / 4} u\right| \leq C_{1}|u|^{1 / 2}\left|A^{1 / 2} u\right|^{1 / 2},\left|A^{3 / 4} u\right| \leq C_{2}\left|A^{1 / 2} u\right|^{1 / 2}|A u|^{1 / 2} .
$$

The foregoing three estimates yields (3.1). Similarly to (3.1) we have

$$
|P(u, \operatorname{grad}) v| \leq C\left|A^{1 / 2} u\right|\left|A^{1 / 2} v\right|^{1 / 2}|A v|^{1 / 2} \quad \text { for } n=3 .
$$

To prove Lemma 3.2 we use Lemma 2.3 and the Sobolev inequalities. To avoid technical difficulties we only give a proof for $p=n, \delta=\theta=1 / 4, \rho=1 / 2$. In this proof we do not distinguish between spaces of vector and scalar functions.

Let $H_{p}^{2 \alpha}$ be the space of Bessel potentials defined in Section 2, i.e., $H_{p}^{2 \alpha}=$ $\left[L_{p}(D), H_{p}^{2}(D)\right]_{\alpha}$. Since the canonical injection $V_{p}^{2 \alpha} \subset H_{p}^{2 \alpha}$ is continuous by Lemma 2.3, the Sobolev inequality implies that the injections 

(a) $V_{n}^{1 / 2} \subset H_{n}^{1 / 2} \subset L_{2 n}$
(b) $V_{2 n / 3}^{1 / 2} \subset H_{2 n / 3}^{1 / 2} \subset L_{n}$

are continuous. The second inclusion (b) yields

$$
\left|A^{-1 / 4} P(u, \operatorname{grad}) v\right|_{n} \leq C|P(u, \operatorname{grad}) v|_{2 n / 3},
$$

since $V_{p}^{1 / 2}$ is equipped with the norm $\left|A^{1 / 4} u\right|_{p}$. Applying the Hölder inequality, we have

$$
|P(u, \operatorname{grad}) v|_{2 n / 3} \leq C_{1}|u|_{2 n}|\operatorname{grad} v|_{n}
$$

since $P$ is continuous from $L_{p}$ to $L_{p}$. The estimates

$$
|u|_{2 n} \leq C_{2}\left|A^{1 / 4} u\right|_{n}, \quad|\operatorname{grad} v|_{n} \leq C_{3}\left|A^{1 / 2} v\right|_{n}
$$

follows from (a) and $V_{p}^{1} \subset H_{p}^{1}$. Combine the above estimates to get the result.

Remark. In Lemma 3.2 we use the same $L_{p}$ spaces in both sides of inequalities. Sometimes this is too restrictive to apply, so we give here a different kind of inequality which is shown just like Lemma 3.2.

$$
|P(u, \operatorname{grad}) v|_{q} \leq M\left|A^{\theta} u\right|_{p}\left|A^{\rho} v\right|_{p}
$$

with $p, q>1, \rho+\theta \geq n(1 / p-1 / 2 q)+1 / 2, \rho+n(1 / q-1 / p) / 2>1 / 2, \theta>0$.

\section{§4. A Priori Estimates}

In this section we derive well known a priori estimates for the solutions of the Navier-Stokes equations.

Suppose that $u=u(x, t)$ is a sufficiently smooth solution of

$$
d u / d t+A u+B u=0 \quad(t>0), \quad u(0)=a .
$$

Here and hereafter we assume, for simplicity, that $P f=0$ and that $u$ is a real vector function.

We first discuss the energy estimate. Multiply $u$ to both sides of (I) and integrate over $D$ to get

$$
(d u / d t, u)+(A u, u)+(B u, u)=0 ;
$$

here $(v, w)$ is the inner product of $H=X_{2}$. Since Lemma 3.1 implies $(B u, u)=0$, this yields

$$
-\frac{1}{2} \frac{d}{d t}|u|^{2}+\left|A^{1 / 2} u\right|^{2}=0 \text {. }
$$

Integrate this over $[0, i]$ to get 


$$
|u|^{2}(t)+2 \int_{0}^{t}\left|A^{1 / 2} u\right|^{2}(s) d s=|a|^{2} .
$$

This is called the energy equality.

We now estimate higher space derivatives of $u$. Multiply $A^{2 \sigma} u(0<\sigma \leq 1 / 2)$ to both sides of (I) and integrate it over $D$ to get

$$
\left(d u / d t, A^{2 \sigma} u\right)+\left(A u, A^{2 \sigma} u\right)+\left(B u, A^{2 \sigma} u\right)=0 .
$$

Since $A$ is self-adjoint on $H$, we have

$$
\frac{1}{2} \frac{d}{d t}\left|A^{\sigma} u\right|^{2}+\left|A^{\sigma+1 / 2} u\right|^{2}+\left(A^{\sigma-1 / 2} B u, A^{\sigma+1 / 2} u\right)=0 .
$$

As we have seen before, estimates for $A^{\sigma-1 / 2} B u$ depends heavily on the space dimension $n$.

Suppose first $n=2$. If $\sigma<1 / 2$, apply Lemma 3.2 with $p=n=2, \delta=1 / 2-\sigma$, $\theta=\sigma, \rho=1 / 2$ to get

$$
\left|A^{\sigma-1 / 2} B u\right| \leq M\left|A^{\sigma} u\right|\left|A^{1 / 2} u\right| .
$$

Using this and the Schwarz inequality, we have

$$
\begin{array}{r}
\left|\left(A^{\sigma-1 / 2} B u, A^{\sigma+1 / 2} u\right)\right| \leq M\left|A^{\sigma} u\right|\left|A^{1 / 2} u\right|\left|A^{\sigma+1 / 2} u\right| \\
\leq \frac{1}{2}\left|A^{\sigma+1 / 2} u\right|^{2}+M^{\prime}\left|A^{\sigma} u\right|^{2}\left|A^{1 / 2} u\right|^{2}
\end{array}
$$

Applying this to (4.2) yields

$$
\frac{1}{2} \frac{d}{d t}\left|A^{\sigma} u\right|^{2}+\frac{1}{2}\left|A^{\sigma+1 / 2} u\right|^{2} \leq M^{\prime}\left|A^{\sigma} u\right|^{2}\left|A^{1 / 2} u\right|^{2} .
$$

In particular, we have

$$
d y / d t \leq 2 M^{\prime}\left|A^{1 / 2} u\right|^{2} y \quad \text { with } \quad y(t)=\left|A^{\sigma} u\right|^{2}(t) .
$$

This together with the Gronwall inequality yields

$$
y(t) \leq y(0)\left(\exp 2 M^{\prime} \int_{0}^{t}\left|A^{1 / 2} u\right|^{2}(s) d s\right) .
$$

On the other hand the energy equality (4.1) implies

$$
2 \int_{0}^{t}\left|A^{1 / 2} u\right|^{2} d s \leq|a|^{2} \quad \text { for all } \quad t \geq 0 .
$$

By this we eventually have a priori estimate

$$
\left|A^{\sigma} u(t)\right|^{2} \leq K \equiv\left|A^{\sigma} a\right|^{2} \exp M^{\prime}|a|^{2} \quad \text { for all } t \geq 0 .
$$


By (4.3) this in turn yields

$$
\int_{0}^{t}\left|A^{\sigma+1 / 2} u\right|^{2} d s \leq M_{1} K|a|^{2} \quad \text { for all } \quad t \geq 0 .
$$

If $\sigma=1 / 2$, we use (3.1) to estimate $B u$. We now get in place of (4.3)

$$
\frac{1}{2} \frac{d}{d t}\left|A^{1 / 2} u\right|^{2}+\frac{1}{2}|A u|^{2} \leq M^{\prime \prime}|u|^{2}\left|A^{1 / 2} u\right|^{4} .
$$

Clearly (4.1) implies that $|u|^{2}(t)$ is dominated by $|a|^{2}$. As in the proof of (4.4) we get

$$
\begin{aligned}
& \left|A^{1 / 2} u\right|^{2} \leq L \equiv\left|A^{1 / 2} a\right|^{2} \exp M^{\prime \prime}|a|^{4}, \\
& \quad \int_{0}^{t}|A u|^{2} d s \leq M_{2} L|a|^{4} \quad \text { for all } t \geq 0 .
\end{aligned}
$$

This argument is found, for example, in Temam [44].

For $n \geq 3$ we have up to now no global boundedness results for $\left|A^{\sigma} u\right|$ corresponding to (4.4) and (4.5). To see the difficulty we study the case $n=3$ and $\sigma=1 / 2$. Using (3.2), we have

$$
\begin{gathered}
\frac{1}{2} \frac{d}{d t}\left|A^{1 / 2} u\right|^{2}+|A u|^{2} \leq C\left|A^{1 / 2} u\right|^{3 / 2}|A u|^{3 / 2} \\
\leq \frac{1}{2}|A u|^{2}+C^{\prime}\left|A^{1 / 2} u\right|^{6}
\end{gathered}
$$

instead of (4.3); here we apply the Young inequality $a b \leq \varepsilon a^{p}+C(\varepsilon) b^{q}, 1 / p+1 / q$ $=1$ with $p=4 / 3$. Put $y(t)=\left|A^{1 / 2} u\right|^{2}(t)$ to get

$$
d y / d t \leq C^{\prime \prime} y^{3} \text {. }
$$

Since every positive solution of $d y / d t=C^{\prime \prime} y^{3}$ blows up, we get no global estimates for $y$ by this method.

\section{$\S 5$. Weak Solutions}

In this section we review the classical existence and uniqueness theorem of weak solutions of (NS).

A vector function $u(x, t)$ is called a weak solution of (NS) on $[0, T)$ if $u$ satisfies

$$
\begin{gathered}
\int_{0}^{T}\{(u, \partial \varphi / \partial t)+(u, \Delta \varphi)+(u,(u, \operatorname{grad}) \varphi)+(f, \varphi)\} d t+(a, \varphi(x, 0))=0 \\
\int_{0}^{T}(u, \operatorname{grad} \psi) d t=0, \quad u=0 \quad \text { on } \quad S \times(0, T)
\end{gathered}
$$


for all $\varphi^{i}, \psi \in C^{\infty}(D \times[0, T)), \operatorname{div} \varphi=0$ such that $\varphi$ and $\psi$ vanish outside a compact set of $D \times[0, T)$. To see that this is a weak form of (NS) we multiply $\varphi$ and $\psi$ to both sides of (NS) and integrate by parts.

We first state the existence result of weak solutions. For simplicity we assume $P f \equiv 0$.

Theorem 5.1 (Leray [27-29] and Hopf [20]). If $a$ is in $H=X_{2}$. Then there is a global weak solution u of (NS) satisfying the energy inequality'

$$
|u|_{2}^{2}(t)+2 \int_{0}^{t}\left|A^{1 / 2} u\right|_{2}^{2}(s) d s \leq|a|_{2}^{2} \quad \text { for } \text { a.e. } t \geq 0 .
$$

Remark. There are many methods to prove this theorem. The most popular one is the Galerkin method due to Hopf [20] and today we can find it in standard literature, Ladyzhenskaya [26] and Temam [43]. In [43] the semi-discritization method is also discussed. It is interesting to sec that the original proof of Leray is different from foregoing methods.

Remark. When we construct a weak solution, we are forced to use weak convergence. This breaks the equality (4.1), so we only get the inequality (5.1) for weak solution unless it is regular.

We skip the proof of Theorem 5.1. We now discuss the uniqueness of Leray-Hopf solutions. The results heavily depend on the space dimension $n$; see Ladyzhenskaya [26], Lions [30]. Lions and Prodi [31] and Serrin [38]. Conceptually, when $n=2$, we can prove the uniqueness of Leray-Hopf solutions; however when $n \geq 3$, we have no proofs nor counterexamples.

To understand this situation it is convenient to recall dimensional analysis of the equations which is introduced by Caffarelli, Kohn and Nirenberg [3]. If $u(x, t)$ and $p(x, t)$ solve (NS) with $f(x, t)$, then for each $\lambda>0$

$$
u_{j}(x, t)=\lambda u\left(\lambda x, \lambda^{2} t\right), \quad p_{\lambda}(x, t)=\lambda^{2} p\left(\lambda x, \lambda^{2} t\right)
$$

also solve (NS) with $f_{\lambda}(x, t)=\lambda^{3} f\left(\lambda x, \lambda^{2} t\right)$ as far as we do not consider the initial and boundary conditions. We describe this property by assigning a scaling dimension to each quantity

$$
x_{i}: 1, \quad u:-1, \quad f:-3, \quad \partial / \partial x_{i}:-1, \quad t: 2, \quad p:-2, \quad c / \partial t:-2
$$

so that each term in the equation has dimension -3 . This is also compatible with assigning a scaling dimension $-2 \alpha$ to the operator $A^{\alpha}$.

The integral $\int|u|_{2}^{2} d x$ has dimension $n-2$ because $2(-1)+n=n-2$. Simi- 
larly, $\iint\left|A^{1 / 2} u\right|_{2}^{2} d x d t$ has dimension $n-2$ because $2(-2)+n+2=n-2$. Eventually, the energy inequality gives two $n-2$ dimensional estimates.

Lemma 5.2 (Uniqueness). Suppose that u is a weak solution of (NS) on $[0, T)$ satisfying $(5.1)$ for $0 \leq t \leq T . \quad$ If

$$
\int_{0}^{T}|u|_{r}^{s} d t
$$

is finite for some $r$ and such that $2 / s+n / r \leq 1, r>n$. Then such a solution u on $[0, T)$ is unique.

In other words if a zero-dimensional integral (5.2) of $u$ is finite then such $u$ is unique. Before we prove this lemma, we consider the case $n=2$. If $n=2$, the energy estimate (5.1) itself is a zero-dimensional estimate, so we get the uniqueness of Leray-Hopf solutions.

Theorem 5.3. If the space dimension $n$ is two, then there is a unique weak solution of (NS) satisfying the energy inequality (5.1).

Proof. It suffices to estimate the integral (5.2) of the weak solution $u$ in Theorem 5.1. Let $r$ and $s$ be a pair of numbers satisfying $2 / r+2 / s=1, r>2$; notice $n=2$. The Sobolev inequality implies

$$
|u|_{r} \leq C\left|A^{\sigma} u\right|_{2}, \quad 1 / r=1 / 2-2 \sigma / n
$$

see Section 2. Using the interpolation inequality, we eventually have

$$
|u|_{r} \leq C^{\prime}\left|A^{1 / 2} u\right|_{2}^{\alpha}|u|_{2}^{\beta}, \alpha=1-2 / r, \alpha+\beta=1 .
$$

Integrate $|u|_{r}^{s}$ over $[0, T]$ to get

$$
\int_{0}^{T}|u|_{r}^{s} d t \leq\left(C^{\prime}\right)^{s} \int_{0}^{T}\left|A^{1 / 2} u\right|_{2}^{\alpha s}|u|_{2}^{\beta s} d t
$$

Applying the Hölder inequality yields

$$
\left(\int_{0}^{T}|u|_{r}^{s} d t\right)^{1 / s} \leq C^{\prime}\left(\int_{0}^{T}\left|A^{1 / 2} u\right|_{2}^{\alpha s} d t\right)^{1 / s} \sup _{0 \leq t \leq T}|u|_{2}^{\beta} .
$$

Since the definition of $s$ shows $\alpha s=2,(5.1)$ now implies the right hand side is finite; thereby the proof is completed.

Proof of Lemma 5.2. The idea of proof given here is essentially the same as in Lions $[30$, p. 84$]$ except that we use the norm defined by $A^{x}$.

It suffices to prove the case $2 / s+n / r=1$ since $D \times(0, T)$ is bounded. Suppose $u$ and $v$ are two solutions of (NS) satisfying the assumption in Lemma 
5.2. We have

$$
d u / d t+A u+B u=0, \quad d v / d t+A v+B v=0 .
$$

Precisely, we have to care the meaning of the above equations; however, we skip it here. Subtract both sides and set $w=u-v$ to get

$$
d w / d t+A w+B u-B v=0 .
$$

Multiplying both sides by $w$ and integrating them over $D$ yields

$$
\frac{d}{d t}|w|_{2}^{2}+2\left|A^{1 / 2} w\right|_{2}^{2}=2 b(w, w, v) ;
$$

the similar argument is found in Section 4. Here we use Lemma 3.1 to get $(B v-B u, w)=b(w, w, v)$.

Let us estimate $b\left(w^{\prime}, w, v\right)$. Let $r^{\prime}$ be the conjugate number of $r$, i.e., $1 / r$ $+1 / r^{\prime}=1$. Apply the Hölder inequality to get

$$
|b(w, w, v)| \leq|P(w, \operatorname{grad}) w|_{\mathbf{r}^{\prime}}|v|_{r} .
$$

If we note Remark in Section 3, we have

$$
|P(w, \operatorname{grad}) w|_{r^{\prime}} \leq M\left|A^{\theta} w\right|_{2}\left|A^{\rho} w\right|_{2}, \rho+\theta=n / 2 r+1 / 2=1 / s^{\prime}, \rho \geq 1 / 2, \theta>0 ;
$$

here $s^{\prime}$ is the conjugate number of $s$. Use the interpolation inequality to dominate the right hand side by a constant multiple of

$$
|w|{ }_{2}^{\beta}\left|A^{1 / 2} w\right|_{2}^{2 / s^{\prime}}, \quad 2 / s^{\prime}+\beta=2, \quad \beta>0,
$$

since $r>n$ implies $1 / s^{\prime}=\rho+\theta<1$. Combine these and apply the Young inequality to get

$$
|b(w, w, v)| \leq C|w|_{2}^{\beta}\left|A^{1 / 2} w\right|_{2}^{2 / s^{\prime}}|v|_{r} \leq \frac{1}{2}\left|A^{1 / 2} w\right|_{2}^{2}+C^{\prime}|v|_{r}^{s}|w|_{2}^{\beta s} .
$$

By (5.3) we see

$$
\frac{d}{d t}|w|_{2}^{2}+\left|A^{1 / 2} w\right|_{2}^{2} \leq 2 C^{\prime}|v|_{r}^{s}|w|_{2}^{2}
$$

since $\beta s=2$. The Gronwall inequality now implies

$$
|w|_{2}^{2}(t) \leq|w|_{2}^{2}(0)\left(\exp \int_{0}^{t} 2 C^{\prime}|v|_{r}^{s}(\tau) d \tau\right)
$$

The assumption on $v$ guarantees that the right hand side is finite for all $t \leq T$. On the other hand $w(0)=a-a=0$, so we get $w(t) \equiv 0$ on $[0, T]$; thereby the proof is completed. 
Remark. To check the above inequalities it is useful to count the scaling dimension of each quantity.

Remark. As we see later, if we had zero or less than zero-dimensional estimates for weak solutions, we could prove not only the uniqueness but also the regularity; see Serrin [37] and Teman [44, p. 41]. For $n=3$ up to now no zero-dimensional estimates for Leray-Hopf solutions are known. Foias, Guillopé and Temam [8] gives new one-dimensional estimates for weak solutions for $n=3$, when the boundary condition is periodic. They prove

$$
\int_{0}^{T}\left|A^{r / 2} u\right|_{2}^{\alpha} r d t
$$

is finite for $r=1,2, \ldots, \alpha_{r}=2 /(2 r-1)$.

\section{§6. Strong Solutions}

In this section we construct more regular solution called strong solution so that the uniqueness holds. However, when $n \geq 3$, there are no global existence results for strong solutions except initial data is sufficiently small.

Usually to construct strong solutions we use the energy estimate; see Kiselev and Ladyzhenskaya [24], Ladyzhenskaya [26], Foias and Temam [9], Heywood [19] and Temam [43]. However, this method needs to assume that initial data $a$ is very regular although the equation (I) is a parabolic equation.

To improve this point we apply analytic semigroup theory (see Lemma 2.2); this idea is due to Kato and Fujita [10, 23] and Sobolevskii [39], and is recently improved by Giga and Miyakawa [16]. Conceptually the results read:

If a zero-dimensional integral of initial data is finite, then there is a unique strong solution of (I) at least locally.

Theorem 6.1 ([16]). Fix $\gamma \geq 0$ such that $n / 2 p-1 / 2 \leq \gamma<1$. Assume that a is in $V_{p}^{2 \gamma}$. Then there is a unique local strong solution $u$ of (I) with the following properties. For some $T>0$,

(i) $u$ is continuous from $[0, T)$ to $V_{p}^{2 \gamma}$,

(ii) $u$ is continuous from $(0, T)$ to $V_{p}^{2 \alpha}$ and $\left|A^{\alpha} u(t)\right|_{p}=o\left(t^{\gamma-\alpha}\right)$ as $t \rightarrow 0$ for some $\alpha, \gamma<\alpha<1$.

Moreover, $u$ is smooth in $\bar{D} \times(0, T)$. If $\left|A^{\gamma} a\right|_{p}$ is small, then $u$ can be extended to a global strong solution. 
Remark. Here 'strong' means that $u(t)$ is strongly differentiable in $t$ with value in $V_{p}^{2 \gamma}$ for all $t, 0<t<T$.

We give here a rough sketch of the proof; see $[10,16,23]$. To avoid technical difficulty we assume $p=n$ and $\gamma=0$. Instead of solving (I) directly we consider its integral form

$$
u(t)=e^{-t A} a-\int_{0}^{t} e^{-(t-s) A} B u(s) d s, t>0 .
$$

We construct approximate solutions of (II) by the iteration scheme

$$
u_{0}(t)=e^{-t A} a, \quad u_{m+1}(t)=u_{0}(t)-\int_{0}^{t} e^{-(t-s) A} B u_{m}(s) d s, m \geq 0 .
$$

We will estimate $\left\|A^{\star} u_{m}\right\|$, where $\|f\|$ denotes the norm of $f$ in $X_{n}$. Lemma 2.2 implies that

$$
\left\|A^{\alpha} e^{-t A} f\right\| \leq C_{\alpha} t^{-\alpha}\|f\|, \quad \alpha \geq 0 .
$$

This yields the estimate

$$
\left\|A^{\alpha} u_{0}(t)\right\| \leq K_{x 0} t^{-\alpha}, \quad \alpha \geq 0,0<t \leq T
$$

with

$$
K_{\alpha 0}=\sup _{0<t \leq T} t^{\alpha}\left\|A^{\alpha} e^{-t A} a\right\| \leq C_{\alpha}\|a\|<\infty, T>0 .
$$

Suppose that for an $m \geq 0 u_{m}(t)$ satisfies

$$
\left\|A^{\alpha} u_{m}(t)\right\| \leq K_{\alpha m} t^{-\alpha} \quad \text { for all } \alpha \geq 0 .
$$

Apply Lemma 3.2 with $\delta=1 / 4, \theta=1 / 4, \beta=1 / 2$ to get

$$
\left\|A^{-\delta} B u_{m}(s)\right\| \leq M K_{\theta m} K_{\rho m} s^{\delta-1} .
$$

Since $\left\|A^{\alpha+\delta} e^{-(t-s) A} f\right\| \leq C_{\alpha+\delta}(t-s)^{-\alpha-\delta}\|f\|$ by Lemma 2.2 , we thus have

$$
\begin{gathered}
\left\|A^{\alpha} u_{m+1}(t)\right\| \leq K_{\alpha 0} t^{-\alpha}+C_{\alpha+\delta} \int_{0}^{t}(t-s)^{-\alpha-\delta}\left\|A^{-\delta} B u_{m}(s)\right\| d s \\
\leq K_{\alpha . m+1} t^{-\alpha} \quad \text { for all } \alpha, \quad 0 \leq \alpha<1-\delta=3 / 4
\end{gathered}
$$

with

$$
K_{\alpha, m+1}=K_{\alpha 0}+C_{\alpha+\delta} M B(1-\delta-\alpha, \delta) K_{\theta m} K_{\rho m}
$$

where $B(a, b)$ is the beta function. This implies that $u_{m}(t)$ is well-defined for each $m \geq 0$ as an element of $C\left([0, T] ; X_{n}\right) \cap C\left((0, T] ; V_{n}^{2 \alpha}\right)$ for all $\alpha, 0 \leq \alpha<3 / 4$. Moreover, $u_{m}(t)$ satisfies 


$$
\left\|A^{\alpha} u_{m}(t)\right\| \leq K_{\alpha m} t^{-\alpha}, 0 \leq \alpha<3 / 4,0<t \leq T .
$$

Put $k_{m}=\max \left\{K_{\theta m}, K_{\rho m}\right\}$ and recall the definition of $K_{x m}$ to get

$$
k_{m+1} \leq k_{0}+C k_{m}^{2}
$$

with a constant $C$ independent of $m$. An elementary calculation shows that if

$$
k_{0}<1 / 4 C
$$

then for each $m \geq 1$, we have

$$
k_{m}<K<1 / 2 C, \quad K_{\alpha, m+1} \leq K_{\alpha 0}+C_{\alpha+\delta} M B(1-\delta-\alpha, \delta) K^{2} \equiv K_{\alpha}
$$

with a constant $K$ independent of $m$. We thus have

$$
\left\|A^{\alpha} u_{m+1}(t)\right\| \leq K_{\alpha} t^{-\alpha}, \quad 0 \leq \alpha<3 / 4,0<t \leq T .
$$

Using again Lemmas 2.2 and 3.2, we can prove, similarly,

$$
\left\|A^{\alpha}\left(u_{m+1}(t)-u_{m}(t)\right)\right\| \leq 2 K C_{\alpha+\delta}(2 C K)^{m-1} B(1-\delta-\alpha, \delta) t^{-\alpha}, 0 \leq \alpha<3 / 4 .
$$

Since $2 C K<1$, this implies $u_{m}(t)$ converges a solution $u(t)$ of (II). According to a standard argument in functional analysis (see [42]), $u(t)$ is eventually a unique strong solution of $(\mathrm{I})$; for the details see $[10,16,23]$.

We now discuss whether (C) is realized. In (C) $k_{0}$ depends on $T$ and $a$, so there are at least two types of sufficient conditions for $(C)$.

$1^{\circ}$. $T$ is fixed and $a$ is taken so that $\|a\|$ is sufficiently small.

$2^{\circ}$. $a$ is fixed and $T$ is sufficiently small.

We first explain $1^{\circ}$. Suppose $\|a\|$ is small, say $\|a\|<1 / 4 C_{\alpha} C$ for $\alpha=1 / 2$, 1/4. Then clearly $k_{0}<1 / 4 C$ for all $T$. This implies that the solution $u(t)$ of (I) exists for all time if $\|a\|<1 / 4 C_{\alpha} C, \alpha=1 / 2,1 / 4$. Namely, there is a global solution of (I) if $\|a\|$ is sufficiently small.

To show $2^{\circ}$ it is enough to prove

$$
t^{\alpha}\left\|A^{\alpha} e^{-t A} a\right\| \longrightarrow 0 \quad(t \rightarrow 0) \quad \text { for } \quad a \in X_{n} .
$$

This follows from Lemma 2.2 with a small trick; see Kato and Fujita $[10,23]$ and Giga [18].

Let $\left[0, T_{*}\right)$ be the maximal interval where the strong solution $u$ exists. If $a$ is in $V_{n}^{2 \sigma}, \sigma>0$, we can estimate $T_{*}$ from below. Indeed, since Lemma 2.2 implies that

$$
t^{\alpha}\left\|A^{\alpha} e^{-t A} a\right\|=t^{\alpha}\left\|A^{\alpha-\sigma} e^{-t A} A^{\sigma} a\right\| \leq t^{\sigma} C_{\alpha-\sigma}\left\|A^{\sigma} a\right\|,
$$

we get 


$$
T_{*}^{\sigma} \geq C^{\prime} / 4 C\left\|A^{\sigma} a\right\|, \quad C^{\prime}=\min \left\{1 / C_{\alpha-\sigma} ; \alpha=1 / 2,1 / 4\right\} .
$$

Note that both sides have dimension $2 \sigma$. Similarly we have

Lemma 6.2. Let $\gamma=n / 2 p-1 / 2 \geq 0$ and let $\sigma>\gamma$. Let $\left[0, T_{*}\right)$ be the maximal interval on which $u$ given in Theorem 6.1 exists. Suppose that a is in $V_{p}^{2 \sigma}$. Then the estimate

$$
T_{*}^{\sigma-\gamma} \geq N /\left|A^{\sigma} a\right|_{p}
$$

holds with a constant $N$ independent of $a$.

Remark. The solution $u$ in Theorem 6.1 is analytic in $t>0$. This follows easily if we replace $t$ by $\operatorname{Re} t$ in the proof, since by Lemmas 2.1 and $2.2 e^{-t A} f$ is analytic in $t>0$ and $\left|A^{\alpha} e^{-t A} f\right|_{p} \leq C_{\alpha}(\operatorname{Re} t)^{-\alpha}|f|_{p}$; see Giga [17] and papers cited there. Foias and Temam [9] discussed the time-analyticity using the energy estimate. However, the proof in [9] seems more complicated than the proof due to semigroup theory [17]. We can also prove $u$ is analytic in space up to boundary if $S$ is analytic; see [17] and papers cited there. In [45] Temam discussed some higher regularity up to $t=0$ and derived compatibility conditions. The semigroup method also works to prove and improve his results. However, we do not give it here.

If $n=2$, the strong solution can be extended globally in time. In other words we have

Lemma 6.3. Suppose that the space dimension $n$ it two. Let $T_{*}$ be as in Lemma 6.2. Then $T_{*}=\infty$.

Proof. We may assume that $a$ is in $V_{2}^{2 \sigma}, 0<\sigma<1 / 2$, since (ii) in Theorem 6.1 shows that $u(t) \in V_{2}^{2 \sigma}$ for positive $t$.

Suppose that $T_{*}$ is finite. Solve (I) with initial data $u\left(t_{0}\right), t_{0} \in\left(0, T_{*}\right)$. Then by Lemma 6.2 the strong solution exists on $\left(t_{0}, T\left(t_{0}\right)+t_{0}\right)$ such that $T\left(t_{0}\right)^{\sigma}$ $\geq N /\left|A^{\sigma} u\left(t_{0}\right)\right|_{2}$. The uniqueness result of Theorem 6.1 shows that this solution agrees with $u(t)$ on $\left(t_{0}, T\left(t_{0}\right)+t_{0}\right) \cap\left(0, T_{*}\right)$. On the other hand (4.4) implies that $\left|A^{\sigma} u\left(t_{0}\right)\right|_{2}$ is bounded on $\left(0, T_{*}\right)$. Thus $T\left(t_{0}\right)$ is estimated from below by a positive constant independent of $t_{0}$. This eventually implies that $u(t)$ can be extended outside $\left[0, T_{*}\right)$ and contradicts the maximality of $T_{*}$; thereby the proof is completed. 


\section{§7. Singularities of Leray-Hopf Solutions}

In this section using strong solutions given in Section 6, we study regularity in time of Leray-Hopf solutions. If $n=2$, the unique Leray-Hopf solution is eventually the strong solution; see $[26,43]$.

Theorem 7.1. Suppose that the space dimension $n$ is two. Let a be in $H$. Then the unique weak solution satisfying (5.1) agrees with the strong solution given in Theorem 6.1 with $p=n=2$ for a.e. $t \geq 0$. Moreover, this solution satisfies the energy equality (4.1).

Proof. Since the strong solution satisfies the energy equality (4.1) it is enough to prove the first assertion.

Lemma 6.3 implies that strong solution exists uniquely for every time interval $[0, T)$. This strong solution is in particular a weak solution on $[0, T)$ satisfying (5.1). Thus, Theorem 5.3 implies that the Leray-Hopf solution agrees with the strong solution on $[0, T)$, since $n=2$. This completes the proof.

In what follows we assume that the space dimension $n$ is three. We will show that Leray-Hopf solutions are locally unique and regular. To do this we recall the uniqueness theorem due to Sather and Serrin; see Serrin [38] and Temam [43, p. 309].

Lemma 7.2. Let $n=3$ and let $a$ be in $H$. Let $u$ and $v$ are weak solutions of (NS) on $[0, T)$ satisfying (5.1). Suppose that

$$
\int_{0}^{T}|v|_{r}^{s} d t<\infty
$$

for some $r$ and $s$ such that $2 / s+n / r \leq 1, r>n$. Then we have $u \equiv v$ a.e. on $[0, T)$.

This differs from Lemma 5.2 because here we do not assume that the integral (5.2) of $u$ is finite. Conceptually, Lemma 7.2 implies that if there is a regular solution on $[0, T)$ then any Leray-Hopf solution agrees with this solution on $[0, T)$. We skip the proof of this lemma. Applying this lemma, we get the local uniqueness and regularity of Leray-Hopf solutions.

Theorem 7.3. Suppose that $a$ is in $X_{p}, p>n=3$. Then, any weak solution $u$ satisfying (5.1) agrees with the strong solution given in Theorem 6.1 with $p>n, \gamma=0$ on $[0, T)$, as far as the latter exists. Moreover, if $|a|_{p}$ is sufficiently 
small, $u$ agrees with the strong solution for a.e. $t \geq 0$.

Proof. By the last part of Theorem 6.1 it suffices to prove the first assertion. If $p>n$ and $\gamma=0$, (i) in Theorem 6.1 implies that the strong solution satisfies (7.1) with $s=\infty, r=p$. Apply Lemma 7.2 to get the result.

Remark. Even if $p=n$ and $\gamma=0$, (i) implies that a zero-dimensional integral of the solution is finite. However, unfortunately this does not imply that the solution satisfies (7.1) because we need $r>n$ in (7.1). This is why we are forced to assume $p>n$ which is not desirable.

Remark. Conceptually, the result corresponding to Theorem 7.3 is found in Temam [43]. However, the assumption on $a$ is more restrictive than ours; so Theorem 7.3 seems to be new. Fabes, Lewis and Riviere [6] constructed a local weak solution satisfying the assumption of Lemma 7.2 with $a \in X_{p}, p>n$, so their solution agrees with ours.

Remark. The results in Theorem 7.3 hold for $n=4$ because the statement of Lemma 7.2 is valid for $n=4$. It may be true even if $n>4$; however, I do not attempt to check it here.

We do not know whether Leray-Hopf solutions are regular for $n \geq 3$. If Leray-Hopf weak solutions develop singularity, it is natural to estimate the size of the singularities. Leray [29] has proved that Lebesgue measure of time singularities is zero. Scheffer [32] has given an estimate of Hausdorff dimension of the singularities, which he improved in [33-35]; for this direction see also Kaniel and Shinbrot [22] and Foias and Temam [9]. Recently, Caffarelli, Kohn and Nirenberg [3] improved the results of Scheffer [34] and proved that space-time singularities of a "suitable weak solution" has one-dimensional Hausdorff measure zero; see also [25]. We will study time singularity only and give proofs in our formulation; see also Temam [44].

Lemma 7.4. Let $n=3$ and let $u$ be a weak solution one $[0, T)$ satisfying (5.1). Then there is a closed set $E$ of Lebesgue measure zero in $(0, T)$ such that $u$ is smooth in $\bar{D} \times((0, T) \backslash E)$.

Proof. Since (5.1) shows that $u$ is in $L_{2}\left((0, T) ; V_{2}^{1}\right), E=\left\{t ;|\operatorname{grad} u|_{2}=\infty\right\}$ is a set of Lebesgue measure zero. For $t \in(0, T) \backslash E$ since $u(t)$ is in $X_{p}, p>n=3$ by the Sobolev inequality, Theorem 7.3 shows that $u$ agrees with the strong solution on $(t, T(t)+t)$ with initial data $u(t)$ for some $T(t)>0$. This implies that 
$E$ is closed and that $u$ is smooth in $\bar{D} \times((0, T) \backslash E)$; thereby, the proof is completed.

Theorem 7.5. Let $n=3$ and let $u$ be a weak solution on $[0, T)$ satisfying (5.1). Then there is a closed set $E \subset(0, T)$ whose 1/2-dimensional Hausdorff measure vanishes, and such that $u$ is smooth in $\bar{D} \times((0, T) \backslash E)$.

Proof. Let $E$ be as in the proof of Lemma 7.4 and let $\left(r_{i}, s_{i}\right), i \in I$ be the connected components of $(0, T) \backslash E$. A result due to Leray [29] is

$$
\sum_{i \in I}\left(s_{i}-r_{i}\right)^{1 / 2}<\infty .
$$

We will prove (7.2). For $t \in\left(r_{i}, s_{i}\right)$ since $u(t)$ is in $V_{2}^{2 \sigma}, 1 / 2 \geq \sigma>\gamma=1 / 4$, we combine Lemma 6.2 (with $\gamma=1 / 4, p=2$ ) and Theorem 7.3 to get that $u$ agrees with the strong solution on $\left(t, T_{*}(t)+t\right)$ with initial data $u(t)$ and that $T_{*}(t)^{\sigma-\gamma}$ $\geq N /\left|A^{\sigma} u(t)\right|_{2}$. This implies

$$
\left(s_{i}-t\right)^{\sigma-r} \geq T_{*}(t)^{\sigma-\gamma} \geq N /\left|A^{\sigma} u(t)\right|_{2} .
$$

Take $\sigma=1 / 2$ to get

$$
N^{2} /\left(s_{i}-t\right)^{1 / 2} \leq\left|A^{1 / 2} u(t)\right|_{2}^{2} .
$$

Integrate this over $\left(r_{i}, s_{i}\right)$ to get

$$
2 N^{2}\left(s_{i}-r_{i}\right)^{1 / 2} \leq \int_{r_{i}}^{s_{i}}\left|A^{1 / 2} u(t)\right|_{2}^{2} d t
$$

We add all these inequalities for $i \in I$. Thus (7.2) follows from (5.1).

The following part is due to Scheffer's clever idea [32]. For every $\varepsilon>0$ we can find by (7.2) a finite part $I_{c}$ of $I$ such that

$$
\sum_{i \notin I_{c}}\left(s_{i}-r_{i}\right) \leq \varepsilon, \sum_{i \notin I_{c}}\left(s_{i}-r_{i}\right)^{1 / 2} \leq \varepsilon .
$$

Obviously, $[0, T] \backslash \cup_{i \in I_{c}}\left(r_{i}, s_{i}\right)$ consists of a finite number of disjoint closed intervals denoted by $B_{j}, j=1, \ldots, N$. Clearly, $\cup_{j=1}^{N} B_{j} \supset E$. Since $\left(r_{i}, s_{i}\right)$ are mutually disjoint, $\left(r_{i}, s_{i}\right), i \notin l_{\varepsilon}$ is included in one and only one $B_{j}$. Denote $I_{j}$ the set of $i$ 's satisfying $B_{j} \supset\left(r_{i}, s_{i}\right)$ so that $I=I_{\varepsilon} \cup \cup_{j=1}^{N} I_{j}$ and $B_{j}=\left(\cup_{i \in I_{j}}\left(r_{i}, s_{i}\right)\right)$ $\cup\left(B_{j} \cap E\right)$. On the other hand since Lemma 7.4 implies that $E$ has Lebesgue measure zero, we have

$$
\operatorname{diam} B_{j}=\sum_{i \in I_{j}}\left(s_{i}-r_{i}\right)
$$

Thus, we get

$$
\operatorname{diam} B_{j} \leq \sum_{i \notin I_{r}}\left(s_{i}-r_{i}\right) \leq \varepsilon
$$

and 


$$
\sum_{j=1}^{N}\left(\operatorname{diam} B_{j}\right)^{1 / 2} \leq \sum_{j=1}^{N}\left(\sum_{i \in I_{j}}\left(s_{i}-r_{i}\right)\right)^{1 / 2} \leq \sum_{i \notin I_{\varepsilon}}\left(s_{i}-r_{i}\right)^{1 / 2} \leq \varepsilon .
$$

Since $\cup_{j=1}^{N} B_{j} \supset E,(7.3)$ and (7.4) imply that $1 / 2$-Hausdorff measure of $E$ is equal to zero; for the definition of Hausdorff measure see Federer [7]. This completes the proof.

\section{References}

[1] Adams, R. A., Sobolev Spaces, 'Academic Press, New York, 1975.

[2] Agmon, S., Douglis, A and Nirenberg, L., Estimates near the boundary for solutions of elliptic partial differential equations satisfying general boundary conditions II, Comm. Pure Appl. Math., 17 (1964), 35-92.

[ 3 ] Caffarelli, L., Kohn, R. and Nirenberg, L., Partial regularity of suitable weak solutions of the Navier-Stokes equations, Comm. Pure Appl. Math., 35 (1982), 771-831.

[4] Calderón, A. P., Intermediate spaces and interpolation, the complex method, Studia Math., 24 (1964), 113-190.

[ 5] Cattabriga, L., Su un problema al contorno relativo al sistema di equazioni di Stokes, Rend. Sem. Mat. Univ. Padova, 31 (1961), 308-340.

[6] Fabes, E. B., Lewis, J. E. and Riviere, N. M., Boundary value problems for the NavierStokes equations, Amer. J. Math., 99 (1977), 626-668.

[ 7 ] Federer, H., Geometric Measure Theory, Springer Verlag, New York, 1969.

[ 8 ] Foias, C., Guillopé, C. and Temam, R., New a priori estimates for Navier-Stokes equations in dimension 3, Comm. in Partial Differential Equations, 6 (1981), 329-359.

[9] Foias, C. and Temam, R., Some analytic and geometric properties of the solutions of the Navier-Stokes equations, J. Math. Pure Appl., 58 (1979), 339-368.

[10] Fujita, H. and Kato, T., On the Navier-Stokes initial value problem I, Arch. Rational Mech. Anal., 16 (1964), 269-315.

[11] Fujita, $\mathrm{H}$. and Morimoto, $\mathrm{H}$., On fractional powers of the Stokes operator, Proc. Japan Acad., 46 (1970), 1141-1143.

[12] Fujiwara, D., On the asymptotic behaviour of the Green operators for elliptic boundary problems and the pure imaginary powers of some second order operators, J. Math. Soc. Japan, 21 (1969), 481-521.

[13] Fujiwara, D. and Morimoto, H., An $L_{r}$-theorem of the Helmholtz decomposition of vector fields, J. Fac. Sci. Univ. Tokyo, Sec. I, 24 (1977), 685-700.

[14] Giga, Y., Analyticity of the semigroup generated by the Stokes operator in $L_{r}$ spaces, Math. Z., 178 (1981), 297-329.

[15] — Domains of fractional powers of the Stokes operator in $L_{r}$ spaces, Arch. Rational Mech. Anal., to appear.

[16] Giga, Y. and Miyakawa, T., Solutions in $L_{r}$ to the Navier-Stokes initial value problem, Arch. Rational Mech. Anal., to appear.

[17] Giga, Y., Time and spatial analyticity of solutions of the Navier-Stokes equations, Comm. in Partial Differential Equations, 8 (1983), 929-948.

[18] _ - The Navier-Stokes initial value problem in $L^{p}$ and related problems, Nonlinear Partial Differential Equations in Applied Science, Proceedings of U. S. -Japan Seminar, Tokyo, 1982, to appear.

[19] Heywood, J. G., The Navier-Stokes equations: On the existence, regularity and decay of solutions, Indiana Univ. Math. J., 29 (1980), 639-681. 
[20] Hopf. E., Über die Anfangwertaufgabe für die hydrohynamischen Grundgleichungen, Math. Nachr., 4 (1951), 213-231.

[21] Itô, S., The existence and the uniqueness of regular solution of non-stationary NavierStokes equation, J. Fac. Sci. Univ. Tokyo, Sec. I, 9 (1961), 103-140.

[22] Kaniel, S. and Shinbrot, M., Smoothness of weak solutions of the Navier-Stokes equations, Arch. Rational Mech. Anal., 24 (1967), 302-324.

[23] Kato, T., and Fujita, H., On the nonstationary Navier-Stokes system, Rend. Sem. Mat. Univ. Padova, 32 (1962), 243-260.

[24] Kiselev, A. A. and Ladyzhenskaya, O. A., On the existence and the uniqueness of the solution of the non-stationary problem for an incompressible viscous fluid, Izv. Akad. Nauk. USSR, 21 (1957), 655-680.

[25] Kohn, R., Partial regularity and the Navier-Stokes equations, Nonlinear Partial Differential Equations in Applied Science, Proceedings of U. S. -Japan Seminar, Tokyo, 1982, to appear.

[26] Ladyzhenskaya, O. A., The Mathematical Theory of Viscous Incompressible Flow, (Gordon and Breach, New York, 1969).

[27] Leray, J., Etude de diverses équations intégrales nonlinéaires et quelque problèmes que pose l'hydrodynamique, J. Math. Pures Appl., 12 (1933), 1-82.

[28] — Essai sur les mouvements plans d'un liquide visqueux que limitent des parois, J. Math. Pures Appl., 13 (1934), 331-418.

[29] - Sur le mouvement d'un liquide visqueux emplissant l'espace, Acta Math., 63 (1934), 193-248.

[30] Lions, J. L., Quelque Methodes de Résolution des Problèmes aux Limites non Lineaires, (Dunod, Paris, 1969).

[31] Lions, J. L. and Prodi, G., Un théorème d'existence et d'unicité dans les équations de Navier-Stokes en dimension 2, C. R. Acad. Sci. Paris, 248 (1959), 3519-3521.

[32] Scheffer, V., Turbulence and Hausdorff dimension, Turbulence and Navier-Stokes Equations, (Lecture Notes in Math., 565 (1976), Springer-Verlag, 94-112).

[33] - Partial regularity of solutions to the Navier-Stokes equations, Pacific $J$. Math., 66 (1976), 535-552.

[34] —_, Hausdorff measure and the Navier-Stokes equations, Commun. Math. Phys., 55 (1977), 97-112.

[35] — The Navier-Stokes equations in space dimension four, Commun. Math. Phys., 61 (1978), 41-68.

[36] Seeley, R., Norms and domains of the complex powers $A_{B}^{z}$, Amer. J. Math., 93 (1971), 299-309.

[37] Serrin, J., On the interior regularity of weak solutions of the Navier-Stokes equations, Arch. Rational Mech. Anal., 9 (1962), 187-195.

[38] _ _ - The initial value problem for the Navier-Stokes equations, Nonlinear Problems, R. E. Langer ed., University of Wisconsin Press, Madison, (1963), 69-98.

[39] Sobolevskii, P. E., On non-stationary equations of hydrodynamics for viscous fluid, Dokl. Akad. Nauk SSSR, 128 (1959), 45-48, in Russian.

[40] Solonnikov, V. A., On estimates of Green's tensors for certain boundary problems, Soviet Math. Dokl., 1 (1960), 128-131.

[41] — Estimates for solutions of nonstationary Navier-Stokes equations, $J$. Soviet Math., 8 (1977), 467-529.

[42] Tanabe, H., Equations of Evolution, Pitman, London, 1979.

[43] Temam, R., Navier-Stokes Equations, North-Holland, Amsterdam, 1977.

[44] — - Navier-Stokes Equations and Nonlinear Functional Analysis, Université de 
Paris-Sud, Orsay, 1980.

[45] - Behaviour at time $t=0$ of the solutions of semi-linear evolution equations, J. Differential Equations, 43 (1982), 73-92.

[46] Vorovich, I. I. and Yudovich, V. I., Stationary flows of incompressible viscous fluids, Mat. Sbornik, 53 (1961), 393-428 (in Russian).

[47] Yosida, K., Functional Analysis, Springer Verlag, Berlin-Heidelberg-New York, 1964. 